\title{
THE MESSAGE UNDERSTANDING CONFERENCES
}

\author{
Beth M. Sundheim \\ Naval Command, Control, and Ocean Surveillance Center \\ RDT\&E Division (NRaD) \\ Decision Support and AI Technology Branch \\ San Diego, CA 92152-7420 \\ sundheim@nosc.mil
}

The Message Understanding Conferences (MUCs) provide a forum for assessing and discussing progress in the field of natural language processing. Each conference is preceded by a formal evaluation of text analysis systems that have been developed to perform a shared task, as designed by the Government in consultation with evaluation participants from the research community.

The evaluations and conferences bring research groups that are supported by major ARPA contracts together with other research groups, who are attracted by the opportunity to work on a well-defined task with Government-furnished materials, to meet with their peers, and to attract attention to their research and development program. Proceedings of the last three conferences have been published in the open literature; two of those conferences were held in the period covered by Tipster Phase I [1,2].

A wide variety of system architectures, processing techniques, and tools have been tried, tested, and refined in the context of the evaluations, spurring progress in the robust processing of naturally, occurring text. To a large extent, it was the promise shown by the technology in the period between 1987 and 1991 that led the Government to include information extraction technology in the Tipster program. The task orientation and evaluation format for MUC were adopted by the TIPSTER program.

Over the years since the first MUC in 1987, the text processing requirements of the evaluation have evolved from analyzing a small number of paragraph-length naval message narratives in a very restricted subject area (MUC-1 in 1987 and MUC-2 in 1989) to analyzing a large number of full-length news articles in much broader areas. MUC-3 (1991) and MUC-4 (1992) required processing of English newswire articles about Latin American terrorism. The MUC-5 evaluation, which ended in July, 1993, was based on the Tipster articles in English and Japanese on joint ventures and microelectronics.
The application which serves as the basis for evaluating the text analysis systems is primarily information extraction, i.e., the identification and formatting of information contained in the news articles. (A secondary task is document detection, since some articles contain no pertinent information whatsoever.) The MUC-5 application tasks were those that had been defined for the Tipster program. The systems developed by the Tipster-funded contractors (BBN, GE/ CMU/NMSU/Brandeis, UMass/Hughes) were evaluated on the basis of their performance in English in both the joint ventures and microelectronics domains; all but the UMass/Hughes system were also evaluated in Japanese in both domains.

[1] Proceedings of the Fourth Message Understanding Conference (MUC-4), June 1992, Morgan Kaufmann Publishers.

[2] Proceedings of the Fifth Message Understanding Conference (MUC-5), August 1993, Morgan Kaufmann Publishers. 